International Journal of Pure and Applied Mathematics

Volume 88 No. 4 2013, 593-601

ISSN: 1311-8080 (printed version); ISSN: 1314-3395 (on-line version)

url: http://www.ijpam.eu

doi: http://dx.doi.org/10.12732/ijpam.v88i4.13

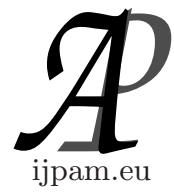

\title{
SPONTANEOUS AMPLIFICATION OF THERMAL NOISE BY A SLACK JOINT
}

${\text { Eugen } \text { Grycko }^{1} \S \text {, Werner Kirsch }}^{2}$, Tobias Mühlenbruch ${ }^{3}$

${ }^{1,2,3}$ Department of Mathematics and Computer Science

University of Hagen

Universitätsstrasse 1, D-58094, Hagen, GERMANY

\begin{abstract}
Recently the phenomenon of enforced thermal noise amplification in a conductor exposed to an electrostatic field has been discovered in a laboratory experiment and qualitatively explained by computations concerning a simplified quantum model. The dispersion of the velocity operator of an electron at thermal equilibrium has turned out to be an appropriate indicator of the thermal noise level inherent in a metal. In the present contribution the behavior of this indicator is studied for an electron confined within a lattice which is interpreted as a quantum position space in the sense of a tight binding model. It turns out that the dispersion of the velocity operator increases when a narrow gap is arranged between two pieces of a metal. An empirical pendant of this computational result is also reported.
\end{abstract}

AMS Subject Classification: 81-04, 81-05, 81V10, 82B10

Key Words: Hamiltonian, quantum Gibbs state, dispersion of the velocity operator

We dedicate this contribution to Professor Andrei Duma from Hagen on the occasion of his $70^{\text {th }}$ birthday.

\section{Introduction}

In [3] and [4] an experimental and a theoretical confirmation of the phenomenon

Received: October 26, 2013

(c) 2013 Academic Publications, Ltd.

$\S_{\text {Correspondence author }}$ url: www.acadpubl.eu 
of thermal noise amplification in a conductor are reported; the conductor is exposed to an electrostatic field which is realized by imposing a high dc voltage on a parallel-plate capacitor. The thermal noise level is measured as the dispersion of the voltage signal between appropriate points of the conductor. This phenomenon is noteworthy insofar as it can be realized by applying only negligible electric power.

In the present contribution we reintroduce a simplified quantum model of a metal. We consider an electron which is sited within a discrete position space and compute the corresponding Hamiltonian (Section 2). This kind of quantum models (tight binding models, cf. [1], p. 168) is subject of intensive explorations (cf. [1], [2] and literature cited therein) and offers an attractive field for computer experimental studies. In Section 3 we reintroduce the quantum Gibbs state describing an electron at thermal equilibrium; an example illustrates the physical plausibility of this simplified description. In Section 4 the quantum dispersion of the velocity operator is defined; in [4] this dispersion is proposed as an indicator of the thermal noise level inherent in a metal. We report on the outcome of a computer experiment whose evaluation suggests thermal noise amplification by a slack joint between two pieces of a metal based on the introduced quantum model. In Section 5 an empirical confirmation of the predicted phenomenon is reported.

\section{A Hamiltonian for an Electron in a Discrete Position Space}

Let us consider a finite lattice

$$
L_{a}:=\{n a \mid n=1, \ldots, N\}
$$

of $N$ points modeling a discrete position space; parameter $a>0$ is called lattice constant. In a simplified tight binding model a quantum state of an electron is described by a function $\varphi: L_{a} \rightarrow \mathbb{C}$ satisfying the condition

$$
\sum_{n=1}^{N}|\varphi(n a)|^{2}=1
$$

In this context $|\varphi(n a)|^{2}$ is interpreted as the probability of spatial association of the electron with lattice point $n a \in L_{a}$. By a standard identification, the set of all electronic states can be viewed as the unit sphere in $\mathbb{C}^{N}$.

The quantum momentum operator $\widehat{p}: \mathbb{C}^{N} \rightarrow \mathbb{C}^{N}$ is defined by

$$
(\widehat{p} \varphi)(n a)=-i \hbar \cdot \frac{\varphi((n+1) a)-\varphi((n-1) a)}{2 a} \quad(n=1, \ldots, N)
$$


where $\hbar$ denotes the (reduced) Planck constant; in (2.1) the convention

$$
\varphi(n a)=0 \text { for } n<1 \text { and for } n>N
$$

is applied and can be interpreted as Dirichlet boundary condition (cf. [2], p. 28ff). $\widehat{p}$ is self-adjoint and serves as a discrete central difference approximation of the 1-dimensional momentum operator

$$
-i \hbar \cdot \frac{d}{d x}
$$

for the position space modeled by the real line. Accordingly, the kinetic energy of an electron is expressed by the operator $\widehat{p}^{2} / 2 m$ where $m$ denotes the electron mass.

Remark 2.1. All entries in the matrix representing operator $\widehat{p}$ are purely imaginary; consequently, all entries in the matrix representing $\widehat{p}^{2} / 2 m$ are real numbers.

Let $1 \leq N_{L 1}<N_{R 1} \leq N_{L 2}<N_{R 2} \leq N$ be four positive integers. Now we introduce potential $U: L_{a} \rightarrow \mathbb{R}$ by

$$
U(n a)= \begin{cases}-e D & \text { for } N_{L 1} \leq n \leq N_{R 1} \\ -e D & \text { for } N_{L 2} \leq n \leq N_{R 2} \\ 0 & \text { elsewhere }\end{cases}
$$

where $-e$ denotes the charge of an electron and $e D$ is interpreted as the depth of potential wells defined in (2.2).

Put

$$
d:=N_{L 2}-N_{R 1} .
$$

If $d=0$, then $U$ has only one potential well whose position is interpreted as the localization of an 1-dimensional piece of a metal placed within $L_{a}$. If $d>0$, then $U$ corresponds to two pieces of a metal separated by a gap of width $d \cdot a$.

To describe an electron confined within lattice $L_{a}$ by a simplified tight binding model we introduce the Hamiltonian

$$
H:=\frac{\widehat{p}^{2}}{2 m}+\widehat{U}
$$

whose potential term $\widehat{U}$ is represented by the (diagonal) matrix

$$
\widehat{U}(n, m)= \begin{cases}U(n a) & \text { if } n=m \\ 0 & \text { if } n \neq m\end{cases}
$$


where $n, m=1, \ldots, N$.

Remark 2.2. All entries in the matrix describing operator $H$ are real numbers.

\section{The Gibbs State of an Electron}

The set of all states of an electron can be embedded into the set of positive semi-definite operators with trace 1 by associating any unit vector $\varphi \in \mathbb{C}^{N}$ with the orthogonal projection onto the 1-dimensional space spanned by $\varphi$. Therefore we call any positive semi-definite operator $Z: \mathbb{C}^{N} \rightarrow \mathbb{C}^{N}$ with trace 1 (generalized) quantum state of an electron sited within lattice $L_{a}$.

Let us consider Hamiltonian $H$ introduced in Section 2. Let $T>0$ denote the temperature of lattice $L_{a}$. The operator $G_{T}: \mathbb{C}^{N} \rightarrow \mathbb{C}^{N}$ modeling the Gibbs state of an electron is given by

$$
G_{T}=\frac{1}{Z(T)} \cdot \exp \left(-\frac{1}{k_{B} \cdot T} \cdot H\right)
$$

where

$$
Z(T):=\operatorname{trace}\left(\exp \left(-\frac{1}{k_{B} \cdot T} \cdot H\right)\right)
$$

denotes the partition function and $k_{B}$ the Boltzmann constant. $G_{T}$ is a positive operator whose trace is equal to 1 . Operator $G_{T}$ is motivated by the entropy principle (cf. [5], p. 384) and describes the thermal equilibrium state of an electron confined within lattice $L_{a}$ with the interpretation of the diagonal entry $G_{T}(n, n)$ as the probability of spatial association of the electron with lattice point $n a$.

Remark 3.1. All entries in the matrix describing operator $G_{T}$ are real numbers; cf. Remark 2.2 .

Example 3.2. Put $N=2000, N_{L 1}=400, N_{R 1}=989, N_{L 2}=1011, N_{R 2}=$ $1600, D=0.1 \mathrm{~V}, T=300 \mathrm{~K}$ and $a=10^{-10} \mathrm{~m}$. Note that $10^{-10} \mathrm{~m}=1 \AA$ corresponds to the typical order of magnitude for the distance between adjacent ions in a metal.

In Figure 1 the horizontal axis corresponds to lattice $L_{a}$. The graph shows the probabilities $G_{T}(n, n)$ of finding an electron at lattice points $n a, n=1, \ldots, N$. The diagram suggests that the electron prefers sites with low potential energy 


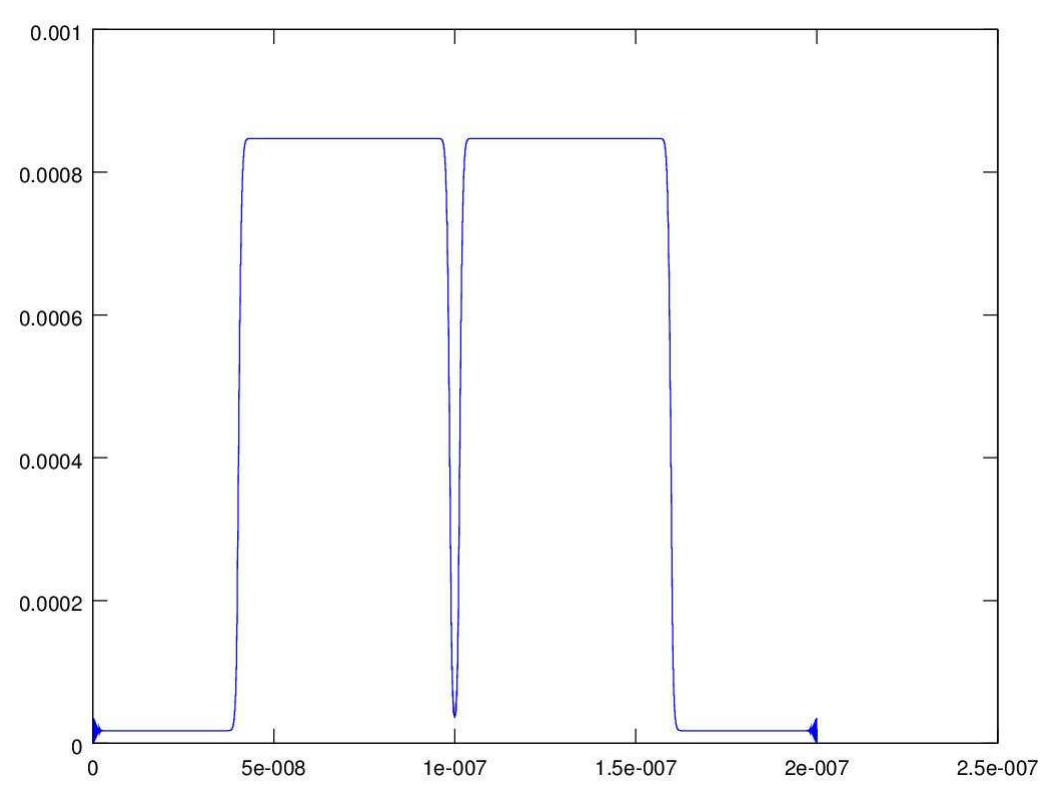

Figure 1: Gibbsian probabilities

w.r.t. potential $U$; an attentive look at Fig. 1 reveals, moreover, that the electron tunnels through the gap between the two pieces of a metal. Both observations underline the physical plausibility of the simplified quantum description of an electron confined within lattice $L_{a}$.

\section{The Dispersion of the Electronic Velocity Operator}

The operator $\widehat{v}: \mathbb{C}^{N} \rightarrow \mathbb{C}^{N}$,

$$
\widehat{v}:=\frac{\widehat{p}}{m},
$$

describes the velocity of an electron sited within lattice $L_{a}$ where $\widehat{p}$ is the momentum operator introduced in Section 2.

The quantum expectation $\mathbb{E}_{\mathrm{q}}(\widehat{v})$ of the velocity of an electron whose state is described by $G_{T}$, is given by

$$
\mathbb{E}_{\mathrm{q}}(\widehat{v})=\operatorname{trace}\left(G_{T} \widehat{v}\right) .
$$


From Linear Algebra it is known that the trace in (4.1) is a real number. Since the matrix describing operator $G_{T}$ is real and the Hermitian matrix corresponding to operator $\widehat{v}$ is purely imaginary, the trace in (4.1) is imaginary; we conclude that

$$
\mathbb{E}_{\mathrm{q}}(\widehat{v})=0
$$

holds for arbitrary $T>0$. This means that there is no direct electronic current in the considered arrangement, which is physically plausible. (4.2) implies, moreover, that the quantum variance $\mathbb{V}_{\mathrm{q}}(\widehat{v})$ of the velocity operator can be computed according to

$$
\mathbb{V}_{\mathrm{q}}(\widehat{v})=\operatorname{trace}\left(G_{T} \widehat{v}^{2}\right)
$$

The quantum mechanical dispersion of velocity operator $\widehat{v}$ is defined by

$$
\mathbb{D}_{\mathrm{q}}(\widehat{v}):=\sqrt{\mathbb{V}_{\mathrm{q}}(\widehat{v})}
$$

this dispersion can be viewed as an indicator of the thermal noise level inherent in the quantum arrangement.

Example 4.1. Put $N=1000, a=10^{-10} \mathrm{~m}, T=300 \mathrm{~K}, D=0.5 \mathrm{~V}$. In a computer experiment we fix the parameters

$$
N_{R 1}-N_{L 1}=N_{R 2}-N_{L 2}=300
$$

of potential $U$ imposed on lattice $L_{a}$ (cf. Section 2); this means that we fix the lengths $L_{1}=L_{2}=3.0 \cdot 10^{-8} \mathrm{~m}$ of two pieces of a metal. The width $d \cdot a$ of the gap between the pieces is varied according to

$$
d=N_{L 2}-N_{R 1}=0,1, \ldots, 160 .
$$

For each choice of $d$ the corresponding dispersion value of the velocity operator is computed; note that in the case $d=0$ the two pieces of metal are joined together and for $d=160$ the maximal width $1.6 \cdot 10^{-8} \mathrm{~m}$ of the gap is attained.

In Figure 2 the horizontal axis corresponds to the width of the gap between two pieces of a metal (the physical unit is $\mathrm{m}$ ) and the vertical axis to the quantum dispersion of the velocity operator (the physical unit is $\mathrm{m} / \mathrm{s}$ ). The diagram shows that the dispersion of electronic velocity increases for increasing width of the gap. Note that the Maxwellian dispersion of thermal velocity of electrons at $T=300 \mathrm{~K}$ is $6.7431 \cdot 10^{4} \mathrm{~m} / \mathrm{s}$. 


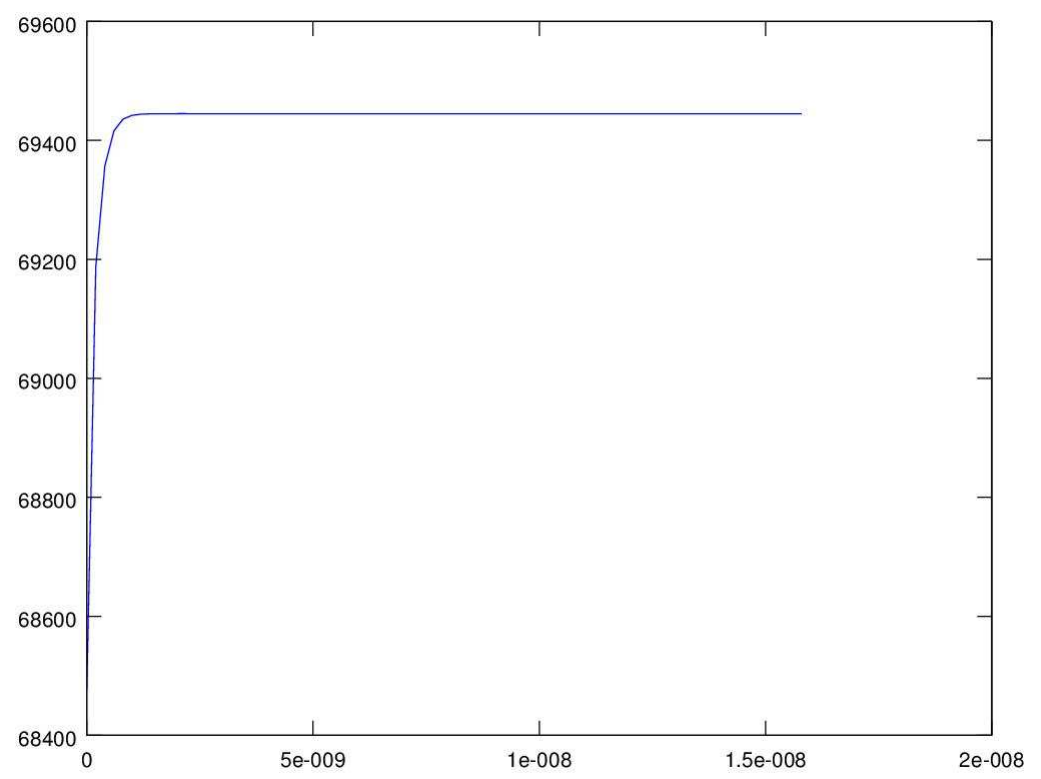

Figure 2: Dispersion of the velocity operator

\section{An Empirical Pendant of the Computational Result}

Example 4.1 suggests that the thermal noise level of electrons positioned within two pieces of a metal should increase if a small gap is arranged between them. Figure 3 shows an aluminium cylinder separated from an aluminium cube by a narrow gap. The thermal voltage signal between the cylinder and the cube is visualized on the screen of an oscilloscope. According to our experience, the measured amplitude of the thermal voltage signal exceeds $10 \mathrm{mV}$ and becomes significantly smaller when the cylinder and the cube are connected. This observation can be interpreted as an empirical pendant of the computer experimental result based on a simplified quantum model and reported in Section 4 .

Remark 5.1. Classical Thermodynamics seems to be unable to explain the phenomenon illustrated in Figure 3. We believe, however, that a quantitative explanation of the amplification of thermal noise by a slack joint between two pieces of a metal would be a challenging objective for Quantum Thermodynamics based on random Hamiltonians. 


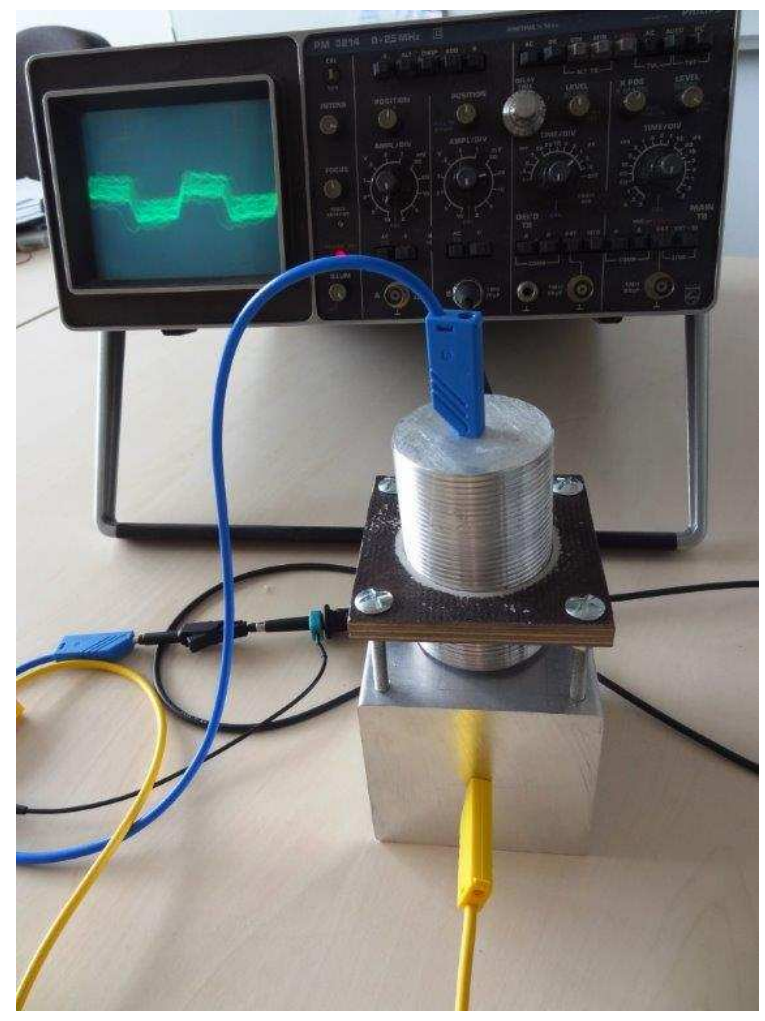

Figure 3: Thermal noise amplification by a slack joint

\section{Acknowledgments}

The authors would like to thank Leonid Pastur from Kharkiv/Ukraine for stimulating discussions on the subject of the contribution.

\section{References}

[1] H.L. Cycon, R.G. Froese, W. Kirsch, B. Simon, Schrödinger operators, Springer, Berlin-Heidelberg-New York (2008).

[2] M. Disertori, W. Kirsch, A. Klein, F. Klopp, V. Rivasseau, Random Schrödinger Operators, Panoramas et Syntheses, Societe Mathematique de France, Paris (2008). 
[3] E. Grycko, W. Kirsch, T. Mühlenbruch, Amplification of thermal noise by an electrostatic field, Int. J. Pure Appl. Math., 61, No. 2 (2010), 187-192.

[4] E. Grycko, W. Kirsch, T. Mühlenbruch, Some quantum mechanical evidence for the amplification of thermal noise in an electrostatic field, Int. J. Pure Appl. Math., 69, No. 4 (2011), 437-443.

[5] W. Thirring, Quantum Mathematical Physics, Second Edition, Springer, Berlin-Heidelberg-New York (2002). 
